\title{
Organic Radical-Linked Covalent Triazine Framework with
}

\section{Paramagnetic Behavior}

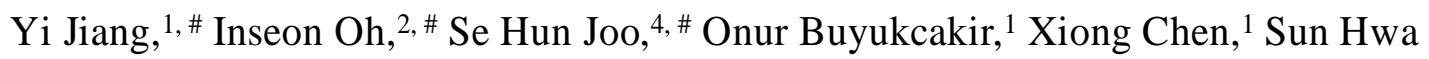

Lee, ${ }^{1}$ Ming Huang, ${ }^{1}$ Won Kyung Seong, ${ }^{1}$ Jin Hoon Kim, ${ }^{3}$ Jan-Uwe Rohde, ${ }^{3}$ Sang Kyu Kwak, ${ }^{4}$ Jung-Woo Yoo, ${ }^{2, *}$ and Rodney S. Ruoff ${ }^{1,2,3,4, *}$

${ }^{1}$ Center for Multidimensional Carbon Materials (CMCM), Institute for Basic Science (IBS), Ulsan 44919, Republic of Korea

${ }^{2}$ School of Materials Science and Engineering, Ulsan National Institute of Science and Technology (UNIST), Ulsan 44919, Republic of Korea

${ }^{3}$ Department of Chemistry Ulsan National Institute of Science and Technology (UNIST), Ulsan 44919, Republic of Korea

${ }^{4}$ School of Energy and Chemical Engineering, Ulsan National Institute of Science and Technology (UNIST), Ulsan 44919, Republic of Korea

\#These authors contributed equally to this work.

*Email: correspondence and requests for materials should be addressed to R.S.R.

(rsruoff@ibs.re.kr; ruofflab@gmail.com), S.K.K. (skkwak@unist.ac.kr ), and J.-W.Y. (jwyoo@unist.ac.kr). 


\section{Synthesis of trialdehyde 1}

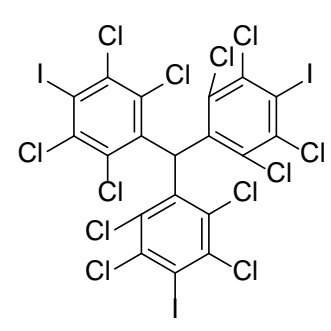

3<smiles>O=Cc1ccc(B(O)O)cc1</smiles>

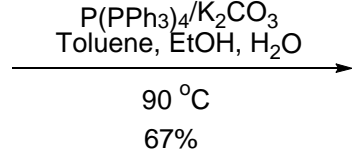

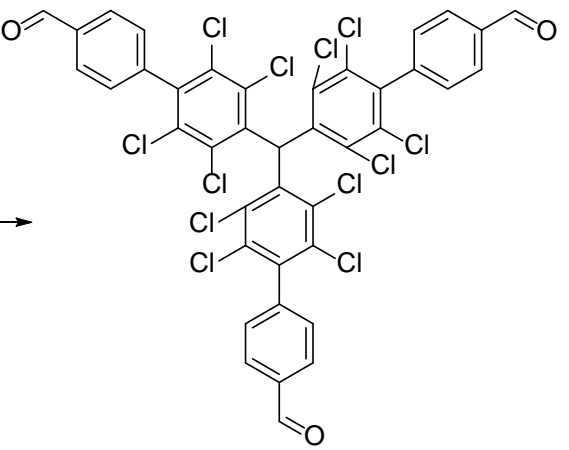

1

A $250 \mathrm{~mL}$ flask containing a magnetic stirrer and a condenser was filled with compound $3^{1}$ (0.9 g, $\left.0.87 \mathrm{mmol}\right), \mathrm{K}_{2} \mathrm{CO}_{3}(0.57 \mathrm{~g}, 4.13 \mathrm{mmol}), \mathrm{Pd}\left(\mathrm{PPh}_{3}\right)_{4}(0.12 \mathrm{~g}, 0.10 \mathrm{mmol}), 4-$ formylphenylboronic acid (600 mg, $4.00 \mathrm{mmol}$ ) and a degassed mixture of toluene (100 $\mathrm{mL}), \mathrm{H}_{2} \mathrm{O}(30 \mathrm{~mL})$ and ethanol $(15 \mathrm{~mL})$ in a $\mathrm{N}_{2}$ atmosphere. The mixture was vigorously stirred at $90{ }^{\circ} \mathrm{C}$ for $36 \mathrm{~h}$. After cooling to room temperature, the solution was extracted with $\mathrm{CH}_{2} \mathrm{Cl}_{2}(50 \mathrm{~mL} \times 3)$, and the organic layer was dried over $\mathrm{Na}_{2} \mathrm{SO}_{4}$. The solvent was then removed under reduced pressure, and the resulting residue was purified by column chromatography on silica gel to produce trialdehyde 1 as a white solid (565 mg, 67 \% yield). ${ }^{1} \mathrm{H}$ NMR (400 MHz, CD $2 \mathrm{Cl}_{2}$ ): $\delta(\mathrm{ppm}) 10.10$ (s, 3H), 8.03 (d, $\left.J=8.4 \mathrm{~Hz}, 6 \mathrm{H}\right), 7.48$ (d, $J=$ $8.4 \mathrm{~Hz}, 6 \mathrm{H}), 7.28$ (s, 1H). ${ }^{13} \mathrm{C}$ NMR (100 MHz, $\left.\mathrm{CD}_{2} \mathrm{Cl}_{2}\right): \delta(\mathrm{ppm})$ 192.0, 143.6, 141.0, 138.5, 136.9, 135.5, 134.5, 134.0, 132.9, 130.4, 130.27, 130.26, 57.4. ESI-MS calculated $\mathrm{m} / \mathrm{z}=968.7[\mathrm{M}-\mathrm{H}]^{-}$, measured $\mathrm{m} / \mathrm{z}=969.0$. 


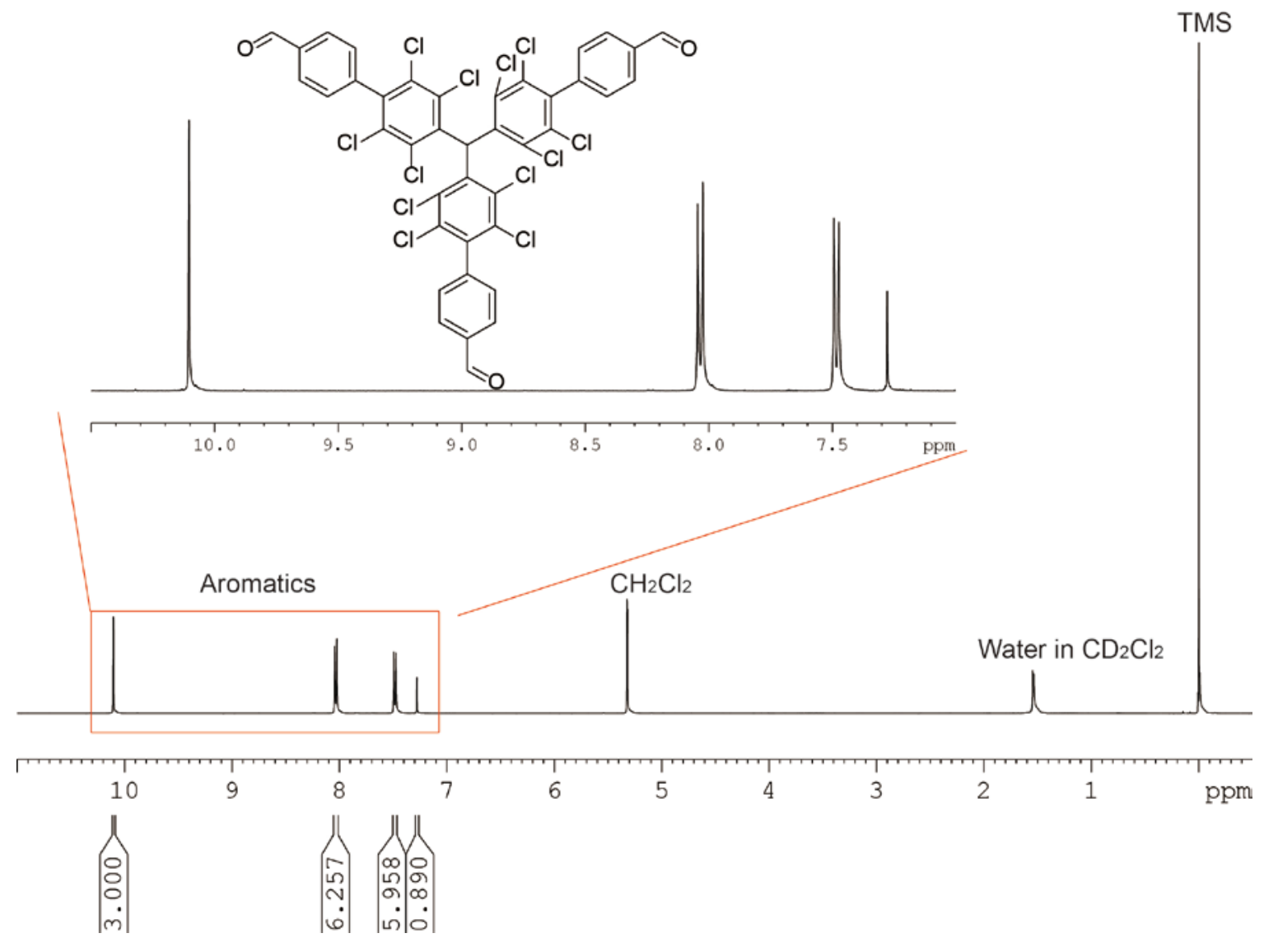

Figure S1. ${ }^{1} \mathrm{H}$ NMR spectrum of trialdehyde $1\left(400 \mathrm{MHz}, \mathrm{CD}_{2} \mathrm{Cl}_{2}\right)$. 


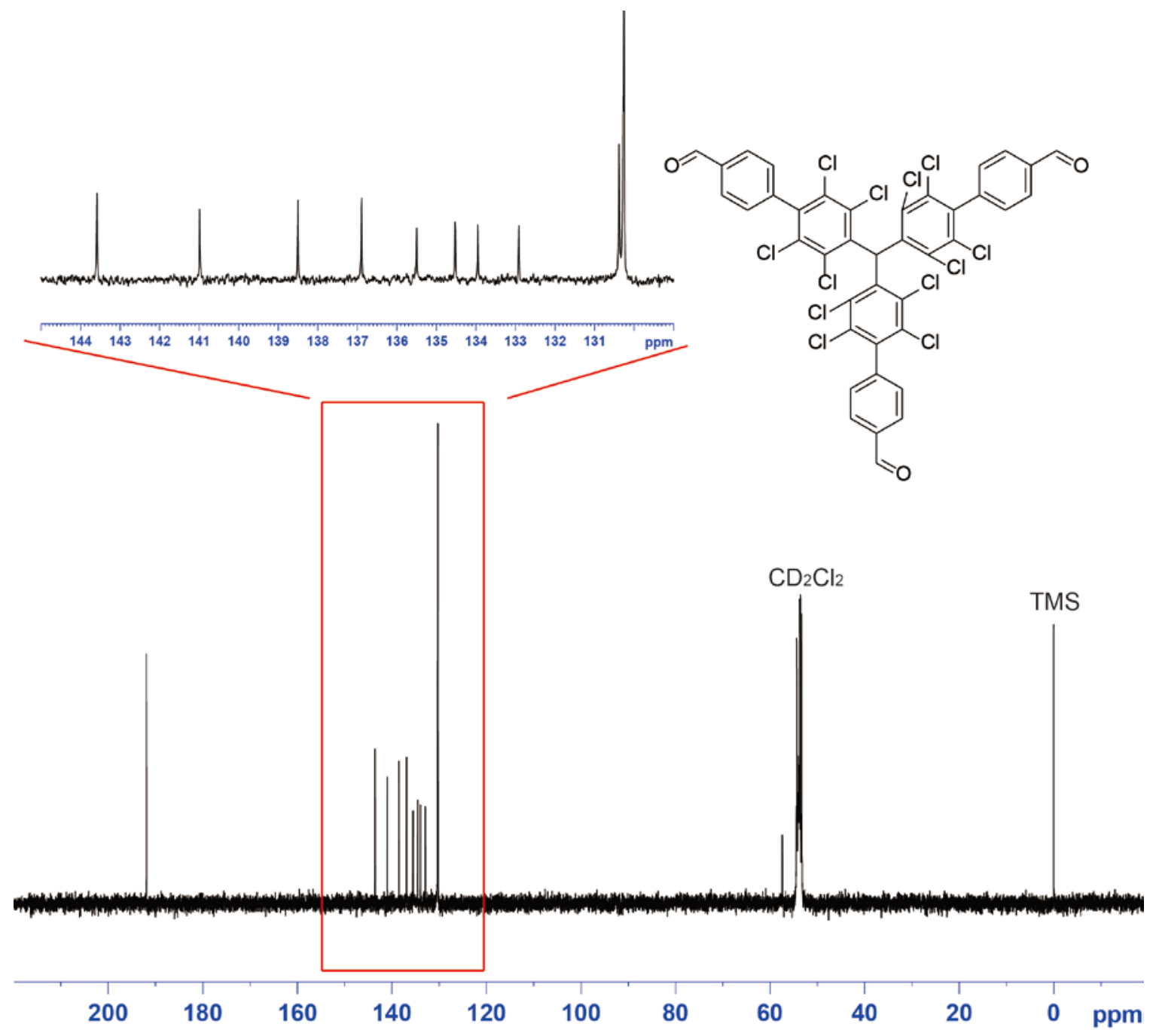

Figure S2. ${ }^{13} \mathrm{C}$ NMR spectrum of trialdehyde $1\left(100 \mathrm{MHz}, \mathrm{CD}_{2} \mathrm{Cl}_{2}\right)$. 
Thermal gravimetric analysis (TGA) of PTMR-CTF.

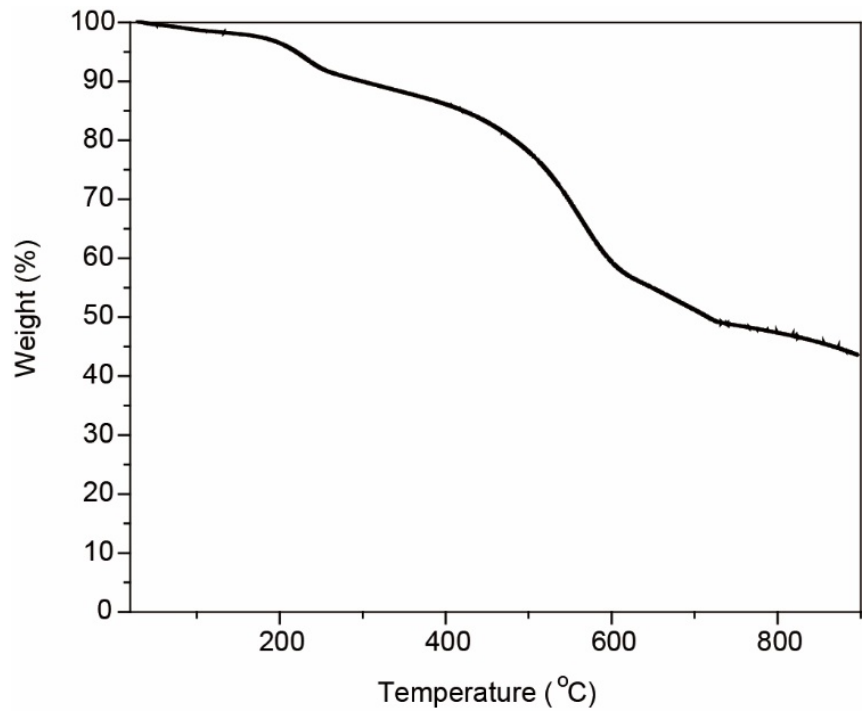

Figure S3. TGA of PTMR-CTF under $\mathrm{N}_{2}$. 
FT-IR spectra of trialdehyde 1, diamidine salt 2, PTM-CTF, and PTMR-CTF.

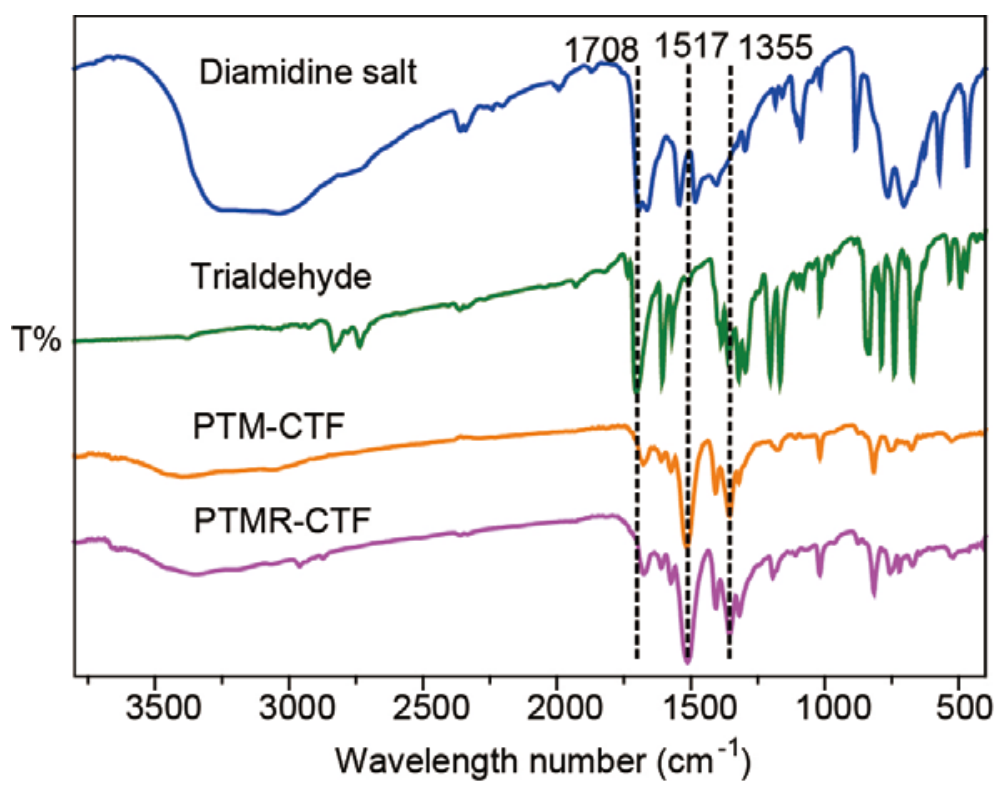

Figure S4. FT-IR spectra of trialdehyde 1, diamidine salt 2, PTM-CTF, and PTMRCTF. 
High-resolution Cl2p XPS spectrum of PTMR-CTF.

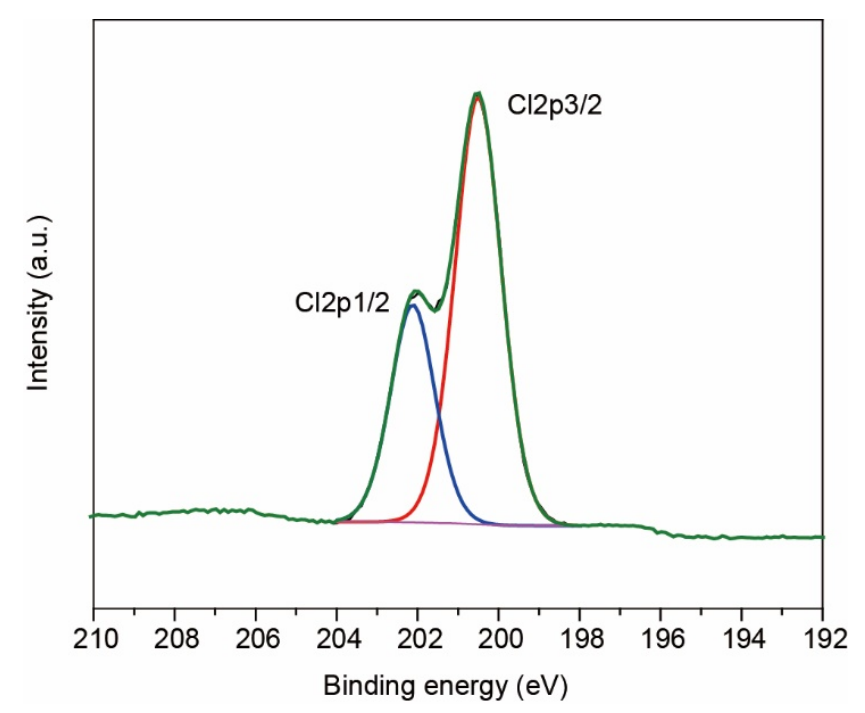

Figure S5. High-resolution Cl2p XPS spectrum of PTMR-CTF. 


\section{Magnetization as a function of the applied field, recorded at $300 \mathrm{~K}$.}

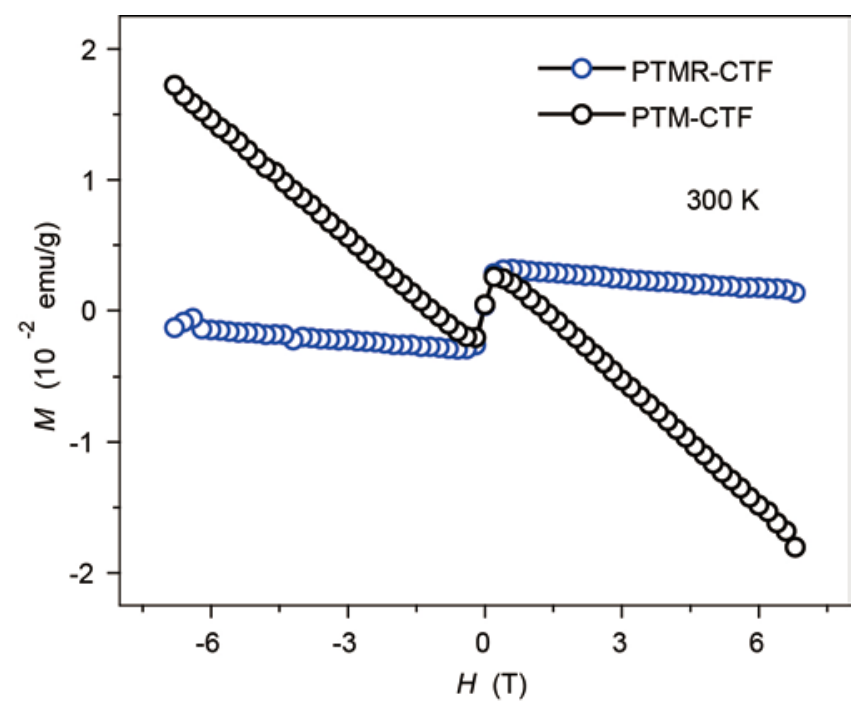

Figure S6. Magnetization as a function of applied field, recorded at 300K. The untreated PTM-CTF sample shows weak ferromagnetism as a result of impurities and diamagnetism. The PTMR-CTF sample also exhibits weak ferromagnetism from impurities and a reduced diamagnetic background due to a paramagnetic contribution. Because both the ferromagnetic and diamagnetic components are nearly temperature independent, we subtracted both components measured for the PTM-CTF sample at $300 \mathrm{~K}$ from all magnetic data recorded for the PTMR-CTF sample. The ferromagnetic impurity in the PTMR-CTF sample was estimated from the saturation value measured at $300 \mathrm{~K}$ and $2 \mathrm{kG}$ $\left(\sim 3.21 \times 10^{-3} \mathrm{emu}^{\cdot} \mathrm{g}^{-1}\right)$. The obtained diamagnetic susceptibility $M / B$ was around $-3.20 \times 10^{-}$ ${ }^{7} \mathrm{emu} \cdot \mathrm{G}^{-1} \cdot \mathrm{g}^{-1}$, which is a reasonable value for typical diamagnetism. The mass of the sample used was about $10 \mathrm{mg}$ (11.4 mg PTM-CTF and $9.4 \mathrm{mg}$ of PTMR-CTF). The sample was kept in a gelatin capsule. The total mass of the diamagnetic background (sample + gelatin capsule) was around $50 \mathrm{mg}$. In order to confirm our procedure of background subtraction, we also measured the diamagnetism of an empty gelatin capsule, which was slightly less 
than that estimated from high field data at $300 \mathrm{~K}$. This discrepancy is caused by the additional mass of carbon and nitrogen in our sample. Thus, throughout the manuscript, we performed diamagnetic background subtraction using high field data at $300 \mathrm{~K}$. 
Determination of the angular momentum quantum number $J$ by a Brillouin function fit.
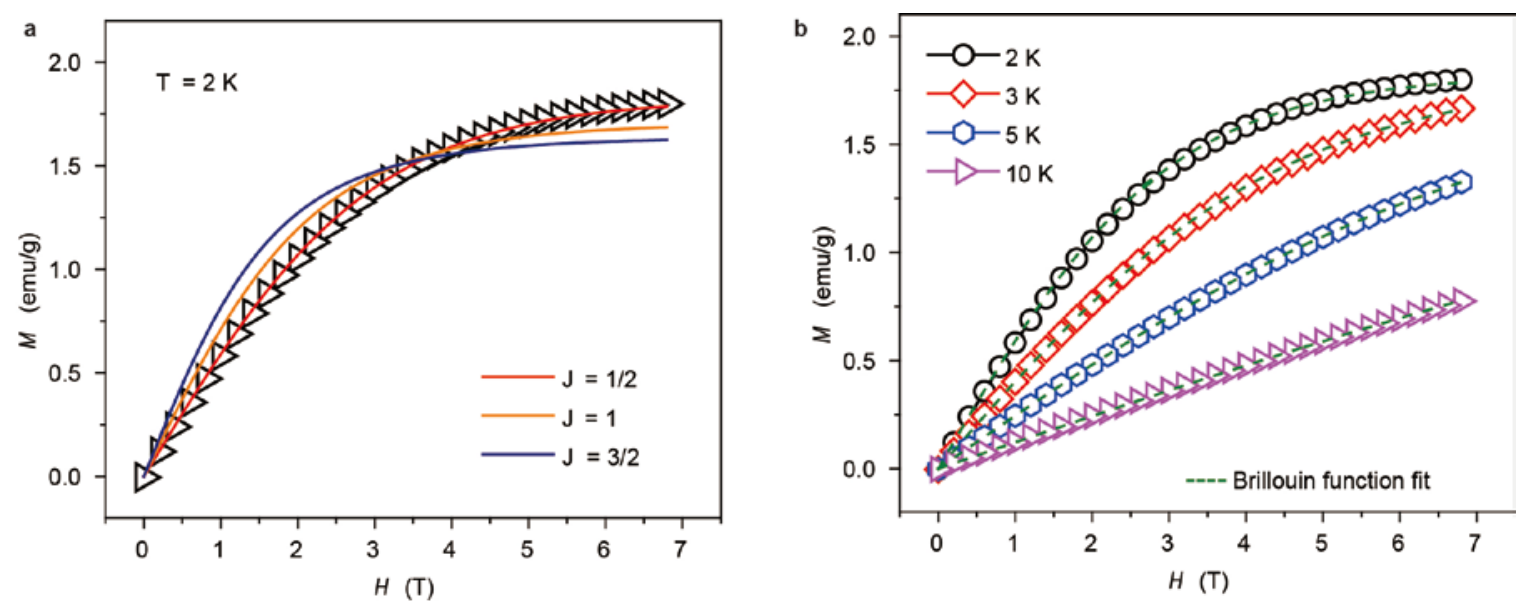

Figure S7. Determination of the angular momentum quantum number $J$ by a Brillouin function fit. a, Magnetization as a function of field $B$ at $2 \mathrm{~K}$ (black symbols). Fits to the Brillouin function with $J=1 / 2,1$, and 3/2 are indicated with solid lines. The $g$ value was assumed to be 2 in the fitting procedure. Only $J=1 / 2$ produces an excellent Brillouin function fit to the measured magnetization. b, Magnetization as a function of $B$ at different temperatures ( $T=2,3,5$, and $10 \mathrm{~K})$. Dotted green curves are fits to the Brillouin function with $J=1 / 2$ and $g=2$. 


\section{Determination of total saturation magnetization.}

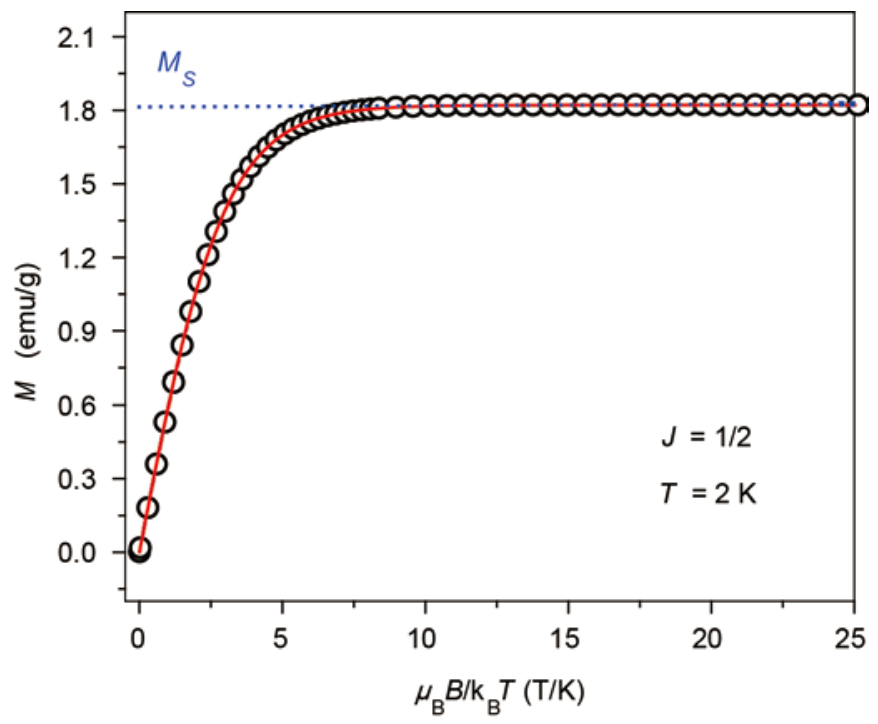

Figure S8. Determination of total saturation magnetization. $M$ vs $H$ plots obtained using parameters obtained from Brillouin function fit to the measured data as shown in Figure S6. The extracted $M_{\mathrm{s}}$ value was $1.82 \mathrm{emu} / \mathrm{g}$. 
Temperature dependence of magnetization obtained with an applied field of $B=50$ mT.

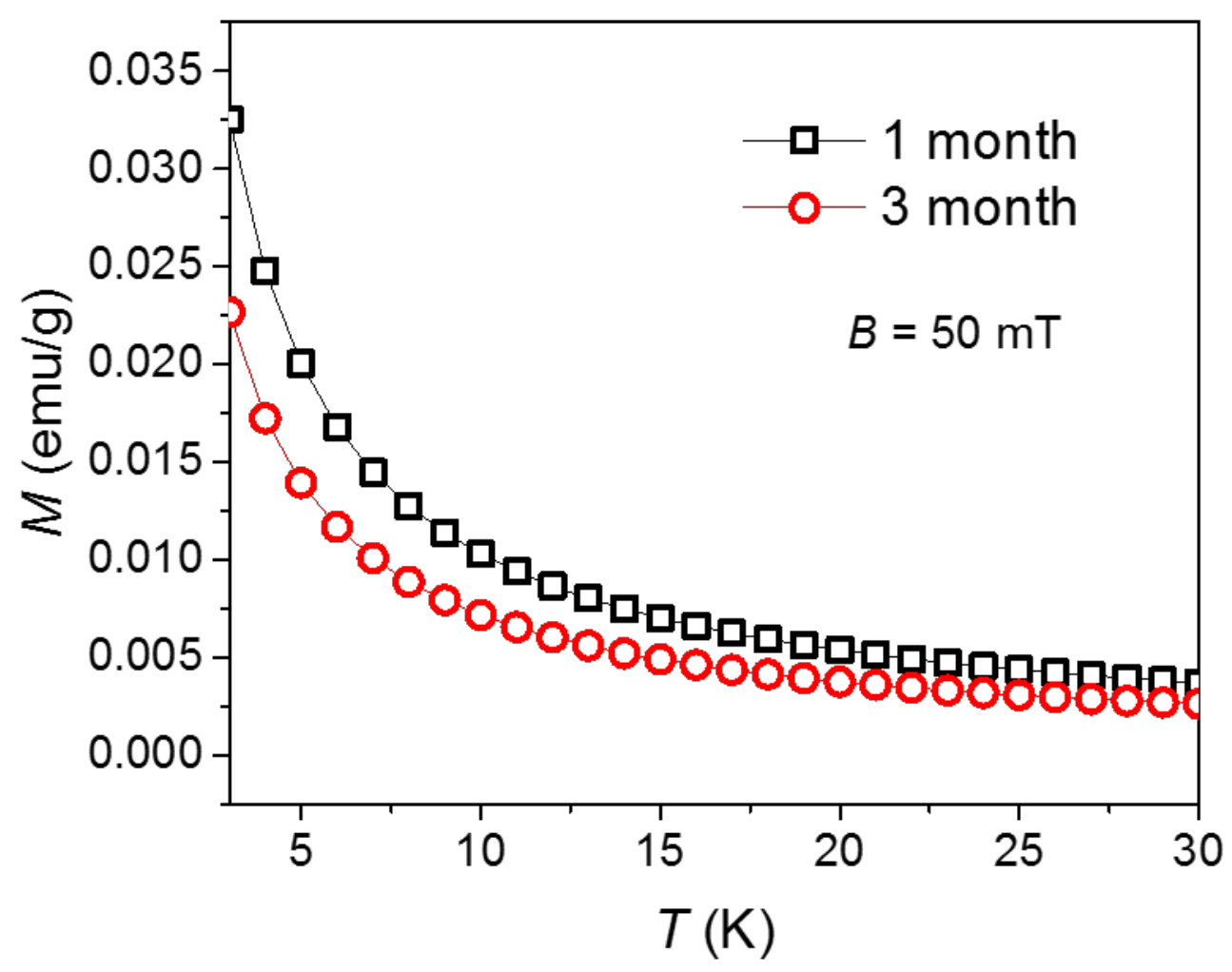

Figure S9. Temperature dependence of magnetization obtained with an applied field of $B=$ $50 \mathrm{mT}$ for PTMR-CTF powder stored in nitrogen after 1 month and 3 months. 
Concentrations of selected metals in PTM-CTF and PTMR-CTF samples, as determined by ICP-MS.

\begin{tabular}{|c|c|c|c|c|c|c|c|}
\hline Sample & Co (ppm) & Li (ppm) & K (ppm) & Mn (ppm) & Ni (ppm) & Fe (ppm) & Cd (ppm) \\
\hline PTM-CTF & 5.31 & - & 23.81 & 19.72 & 11.76 & 32.13 & 0.03 \\
\hline PTMR-CTF & 6.10 & 1.12 & 21.42 & 16.73 & 9.67 & 29.35 & 0.06 \\
\hline
\end{tabular}




\section{Details of density functional theory calculations}

Spin-polarized density functional theory (DFT) calculations were performed using the DMol3 module. ${ }^{2-4}$ The generalized gradient approximation with the Perdew-BurkeEnzerhof functional (GGA-PBE) ${ }^{5}$ was used to describe the exchange-correlation potential of the electrons. Core electrons were treated as all electrons explicitly with relativistic effects. The long-range van der Waals interactions were corrected using Grimme's method. ${ }^{6}$ The molecular orbitals were expanded using the numerical atomic orbital basis set at the level of DNP 4.4. The Brillouin zone was sampled by a $1 \times 1 \times 1 k$-point set. A selfconsistent field calculation was performed with the convergence criterion of $1.0 \times 10^{-6}$ and the global orbital cutoff of $3.8 \AA$. The convergence criteria for the geometry optimization were set to $1.0 \times 10^{-5} \mathrm{Ha}$ for the maximum energy change, $0.002 \mathrm{Ha} / \AA$ for the maximum force, and $0.005 \AA$ for the maximum displacement. Periodic boundary conditions were applied in three dimensions except for the calculation of small-molecule PTMR derivatives. To minimize the interactions between periodic images, a vacuum slab thicker than $15 \AA$ was added to the top of the single layer PTMR-CTF. The spin associated with each atom was computed based on the Mulliken population analysis. ${ }^{7,8}$ 


\section{The optimized structures and calculated spin densities of molecular PTMR derivatives.}

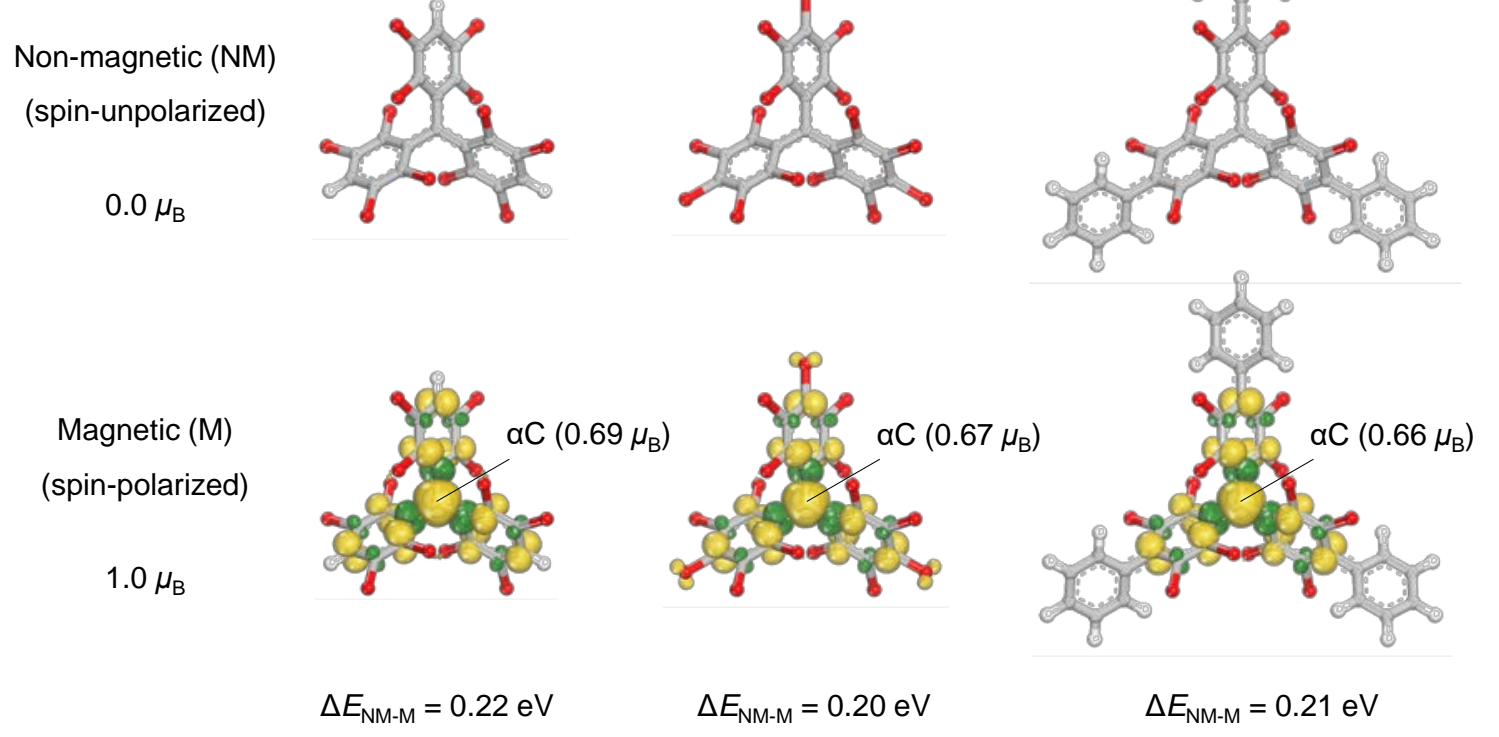

Figure S10. The optimized structures and calculated spin densities of molecular PTMR derivatives. The iso-surface of the spin density is represented with the iso-value of 0.009 electrons $/ \AA^{3}$. The yellow and green iso-surfaces indicate the spin densities for $\alpha$-spin and $\beta$-spin electrons, respectively. The stabilities of the non-magnetic and magnetic states of the PTMR derivatives were compared through the spin-unpolarized and spin-polarized calculations. Carbon, chlorine, and hydrogen atoms are light gray, red, and white, respectively. 
The calculated band structure, projected density of states (PDOS), and singly occupied/unoccupied molecular orbitals (SOMOs/SUMOs) of the PTMR-CTF with ferromagnetic (top) and antiferromagnetic (bottom) orderings.
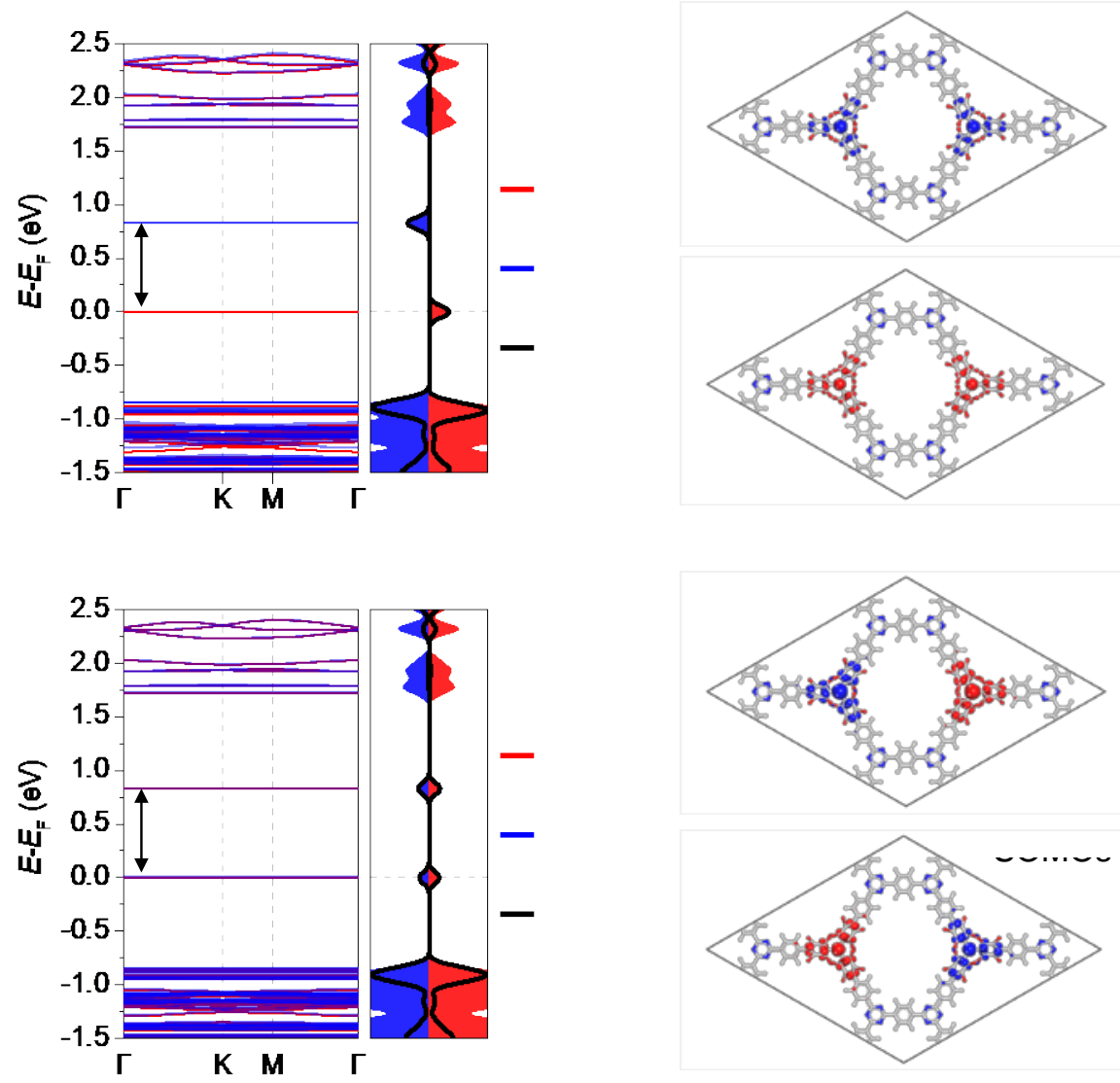

Figure S11. The calculated band structure, projected density of states (PDOS), and singly occupied/unoccupied molecular orbitals (SOMOs/SUMOs) of the PTMR-CTF with ferromagnetic (top) and antiferromagnetic (bottom) ordering. The PDOS shows the total contribution from $s$ and $p$ orbitals for all atoms (red and blue) and PTMR building blocks (black). The iso-surface of the molecular orbital is represented with an iso-value of 0.03 electrons/ $\AA^{3}$. 
The calculated spin densities and the relative energies of the PTMR-CTF with ferromagnetic and antiferromagnetic orderings.
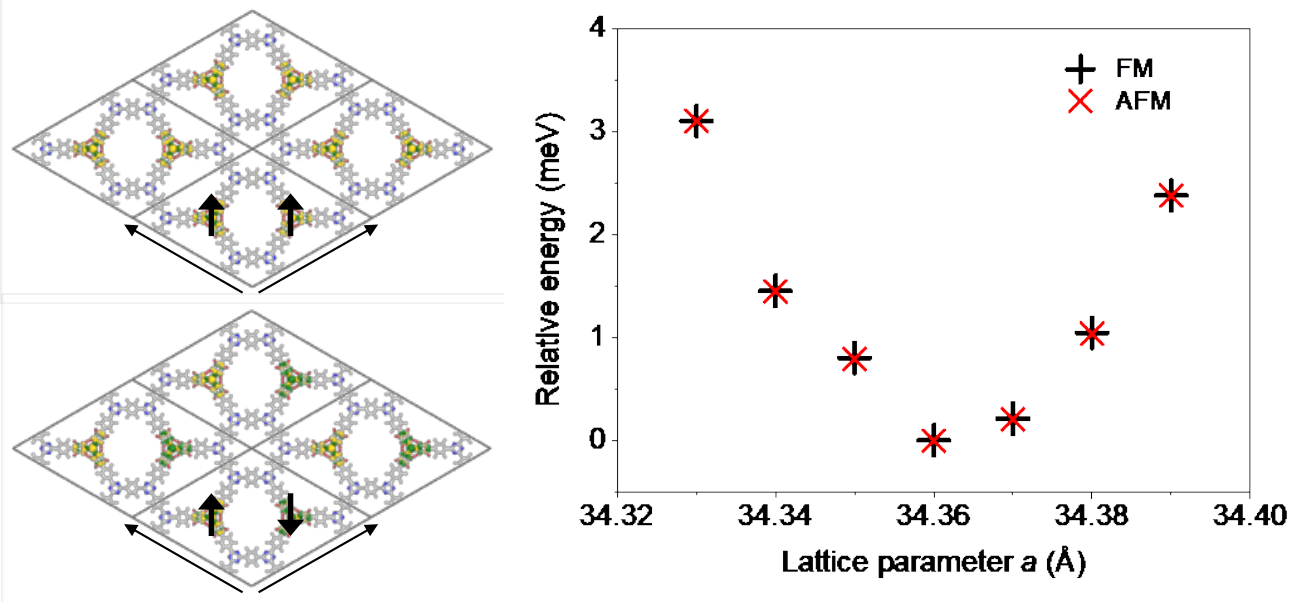

Figure S12. The calculated spin densities (left) and the relative energies (right) of PTMRCTF with ferromagnetic and antiferromagnetic orderings. Note that the lattice parameters $a$ and $b$ were artificially increased and decreased with geometry optimization. The isosurface of the spin density is represented with an iso-value of 0.009 electrons $/ \AA^{3}$. The yellow and green iso-surfaces indicate the spin densities for $\alpha$-spin and $\beta$-spin electrons, respectively. 


\section{References}

(1) Wu, X.; Kim, J. O.; Medina, S.; Ramírez, F. J.; Burrezo, P. M.; Wu, S.; Lim, Z. L.; Lambert, C.; Casado, J.; Kim, D.; Wu, J. Push-Pull-Type Polychlorotriphenylmethyl Radicals: New Two-Photon Absorbers and Dyes for Generation of Photo-Charges. Chem. Eur. J. 2017, 23, 7698-7702.

(2) Delley, B. From Molecules to Solids with the DMol ${ }^{3}$ Approach. J. Chem. Phys. 2000, 113, 77567764.

(3) Delley, B. An All-Electron Numerical Method for Solving the Local Density Functional for Polyatomic Molecules. J. Chem. Phys. 1990, 92, 508-517.

(4) Dassault Systèmes BIOVIA, Materials Studio, 2018 (San Diego: Dassault Systèmes, 2018).

(5) Perdew, J. P.; Burke, K.; Ernzerhof, M. Generalized Gradient Approximation Made Simple. Phys. Rev. Lett. 1996, 77, 3865-3868.

(6) Grimme, S. Semiempirical GGA-Type Density Functional Constructed with a Long-Range Dispersion Correction. J. Comput. Chem. 2006, 27, 1787-1799.

(7) Mulliken, R. S. Electronic Population Analysis on LCAO-MO Molecular Wave Functions. I. J. Chem. Phys. 1955, 23, 1833-1840.

(8) Mulliken, R. S. Electronic Population Analysis on LCAO-MO Molecular Wave Functions. II. Overlap Populations, Bond Orders, and Covalent Bond Energies. J. Chem. Phys. 1955, 23, 18411846. 